\title{
Interactive comment on "Simulating oceanic radiocarbon with the FAMOUS GCM: implications for its use as a proxy for ventilation and carbon uptake” by Jennifer E. Dentith et al.
}

Anonymous Referee \#2

Received and published: 30 November 2019

See attached pdf file

Please also note the supplement to this comment:

https://www.biogeosciences-discuss.net/bg-2019-365/bg-2019-365-RC2-

supplement.pdf

Interactive comment on Biogeosciences Discuss., https://doi.org/10.5194/bg-2019-365, 2019. 